\title{
Einstein's Recoiling Slit Experiment, Complementarity and Uncertainty
}

\author{
Tabish Qureshi ${ }^{1}$ \& Radhika Vathsan ${ }^{2}$ \\ ${ }^{1}$ Centre for Theoretical Physics, Jamia Millia Islamia, New Delhi, India. E-mail: tabish@ctp-jamia.res.in \\ ${ }^{2}$ Department of Physics, BITS Pilani K K Birla Goa Campus, Zuarinagar, Goa, India. E-mail: radhika@goa.bits-pilani.ac.in
}

Editors: Christoph Lehner, Danko Georgiev \& Juan Ferret

Article history: Submitted on November 29, 2012; Accepted on March 24, 2013; Published on May 15, 2013.

W e analyze Einstein's recoiling slit experiment and point out that the inevitable entanglement between the particle and the recoiling slit was not part of Bohr's reply. We show that if this entanglement is taken into account, one can provide a simpler answer to Einstein. We also derive the Englert-Greenberger-Yasin duality relation from the entanglement between the particle and the recoiling slit. In addition, we show that the Englert-Greenberger-Yasin duality relation can also be thought of as a consequence of the sum uncertainty relation for certain observables of the recoiling slit. Thus, the uncertainty relations and entanglement are both an integral part of the which-way detection process.

Quanta 2013; 2: 58-65.

\section{Introduction}

The two-slit experiment carried out with particles is a testbed of various foundational ideas in quantum theory. It has been used to exemplify wave-particle duality and Bohr's complementarity principle [1]. The two-slit ex-

(c) (1) This is an open access article distributed under the terms of the Creative Commons Attribution License CC-BY-3.0, which permits unrestricted use, distribution, and reproduction in any medium, provided the original author and source are credited. periment captures the essence of quantum theory in such a fundamental way that Feynman went to the extent of stating that it is a phenomenon "which has in it the heart of quantum mechanics; in reality it contains the only mystery" of the theory [2].

Neils Bohr had stressed that the wave-nature of particles, characterized by interference, and the particle-nature, characterized by the knowledge of which slit the particle passed through, are mutually exclusive. He argued that in a single experiment, one could see only one of these two complementary properties at a time. Bohr elevated this concept to the status of a separate principle, the principle of complementarity [1].

Einstein was uncomfortable with the quantum indeterminism and sought to demonstrate that the principle of indeterminacy could be violated. Einstein's ongoing criticism of Bohr's view of quantum theory was brought into focus at the fifth Solvay conference in Brussels in 1927. Einstein pointed out how it was possible to use the laws of conservation of energy and momentum to obtain information on the state of a particle in a process of interference which, according to the principle of complementarity, should not be accessible. In the following, we describe Einstein's proposed thought experiment [3]. 


\section{Recoiling Slit Experiment}

Light traveling along the $z$-axis, perpendicular to the $x$ axis, is incident on screen 1 (see Figure 1) which has a slit narrower than the wavelength of light. The light passes through the single slit and illuminates screen 2 which has two slits. Light emerging from the double-slit results in the formation of an interference pattern on the final screen 3. Einstein suggested that screen 1 be free to slide along the $x$-axis. According to his argument, the deflection of the light from the original direction of propagation can only be caused by its interaction with this screen. By the law of conservation of momentum, if the incident particle is deflected towards the top, the screen will recoil towards the bottom and vice-versa. Einstein contended that by measuring the recoil momentum of screen 1 , it is in principle possible to determine which slit each particle passed through. Successive light particles would eventually build up an interference pattern. Einstein argued that such an experiment would show a violation of the principle of complementarity.

Bohr responded to Einstein's experiment by pointing out some subtleties involved in obtaining the which-path information. According to Bohr, to obtain knowledge of which slit the particle had passed through, it was necessary to measure the movement of the screen to a certain degree of accuracy. Any lesser degree of accuracy in the measurement will fall short of providing the which-path information. Consequently, screen 1 should be so sensitive that it should be treated like a quantum object. In order that the recoiling screen gives a well-defined value of the momentum, its initial momentum should be known to a good accuracy. However, due to the uncertainty principle, there will be a degree of uncertainty as to the position of the slit if the momentum of the screen is welldefined. The uncertainty in the position of the slits would lead to a superposition of several slightly shifted patterns, sufficient to eliminate the interference pattern [3].

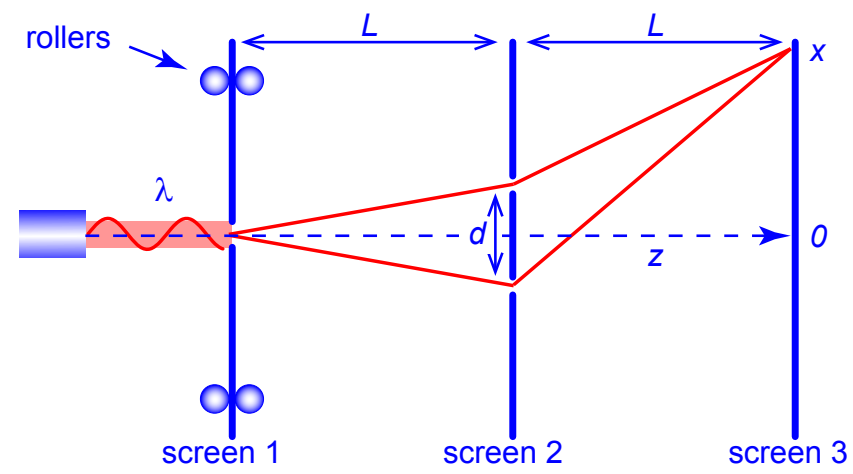

Figure 1: Schematic diagram to illustrate Einstein's recoiling slit experiment.
Wooters and Zurek carried out a quantitative analysis of Bohr's argument, assuming the recoiling slit to be constrained by a harmonic oscillator potential [4]. The experiment was realized in a very interesting manner by the group of Serge Haroche who has been awarded the Nobel Prize in physics for 2012 [5]. They were able to set up a recoiling slit which could be tuned continuously from being quantum-mechanical to being classical. Utter and Feagin realized the experiment by using a trapped ion in place of the recoiling slit [6].

\section{Theoretical Analysis}

\subsection{Which-path information and entanglement}

Bohr's reply to Einstein's recoiling slit experiment relies on the assumption that the recoil experienced by the single slit will necessarily disturb the state of the particle. This disturbance would be just enough to wash out the interference. However, there is a crucial aspect of getting which-path information, which is not part of Bohr's reply. According to von Neumann, a quantum measurement consists of two processes [7]. The first one is a unitary evolution which takes the product state of the system and detector to an entangled state, which establishes correlation between states of the system and those of the detector. For example, if the system is initially in a state $\sum_{i=1}^{n} c_{i}\left|\psi_{i}\right\rangle$ and the detector is in a state $\left|d_{0}\right\rangle$, the first process has the following effect

$$
\left|d_{0}\right\rangle \sum_{i=1}^{n} c_{i}\left|\psi_{i}\right\rangle \stackrel{\text { Unitary evolution }}{\longrightarrow} \sum_{i=1}^{n} c_{i}\left|d_{i}\right\rangle\left|\psi_{i}\right\rangle
$$

The second process is essentially a non-unitary one which picks out a single result (say) $\left|d_{k}\right\rangle\left|\psi_{k}\right\rangle$, from the superposition on the right hand side of the above, with a probability $\left|c_{k}\right|^{2}$. How such a superpostion of possibilities go over to a single outcome in a measurement, is what constitutes the heart of the measurement problem. Here we will only be concerned with the first process. If we apply the preceding argument to the case of Einstein's recoiling slit experiment, there will be two orthogonal states of the particle, $\left|\psi_{1}\right\rangle$ and $\left|\psi_{2}\right\rangle$, and two momentum states of the recoiling slit, $\left|d_{1}\right\rangle$ and $\left|d_{2}\right\rangle$. There are two points to be noted here.

(a) Two different momentum states of the recoiling slit will necessarily get entangled with the states of the particle passing through the two slits.

(b) In principle it is possible to find an interaction which will not affect the states of the particle $\left|\psi_{1}\right\rangle$ and $\left|\psi_{2}\right\rangle$, but will only result in the detector states getting correlated with them. 
Point (a) is something which was not part of Bohr's reply. It can be easily shown that this point alone is enough to rule out any interference pattern for the particle. The combined state of the recoiling slit and the particle, on reaching the screen 3 after passing through the doubleslit, will be of the form

$$
\Psi(x, t)=c_{1}\left|d_{1}\right\rangle \psi_{1}(x, t)+c_{2}\left|d_{2}\right\rangle \psi_{2}(x, t)
$$

The probability of finding the particle at a point $x$ on the screen 3 is given by

$$
\begin{aligned}
& |\Psi(x, t)|^{2}=\left|c_{1}\right|^{2}\left\langle d_{1} \mid d_{1}\right\rangle\left|\psi_{1}(x)\right|^{2}+\left|c_{2}\right|^{2}\left\langle d_{2} \mid d_{2}\right\rangle\left|\psi_{2}(x)\right|^{2} \\
& +c_{1}^{*} c_{2}\left\langle d_{1} \mid d_{2}\right\rangle \psi_{1}^{*}(x) \psi_{2}(x)+c_{2}^{*} c_{1}\left\langle d_{2} \mid d_{1}\right\rangle \psi_{2}^{*}(x) \psi_{1}(x)
\end{aligned}
$$

The last two terms in the above denote interference, and will vanish if the two states of the recoiling slit, $\left|d_{1}\right\rangle$ and $\left|d_{2}\right\rangle$ are distinguishable, i.e., orthogonal to each other. Thus, the very fact that which-path information is carried by the recoiling slit is enough to rule out interference. One need not invoke the position-momentum uncertainty of the recoiling slit. This will happen irrespective of the method one uses to get which-path information in any other variant of this experiment. The argument here is on the lines of the treatment of a particle going through the double-slit interacting with a 1-bit detector by Scully, Englert and Walther [8].

If the inevitable entanglement in the measurement process, and its implications are taken into account, one can provide a rebuttal to Einstein without invoking the position-momentum uncertainty of the recoiling slit. Bohr could not have based his 1927 argument on entanglement between the particle and the recoiling slit, however, because the concept of entanglement was introduced several years later, in 1935, by Erwin Schrödinger [9].

\subsection{Path-distinguishability and fringe visibility}

Let us now look at the more interesting situation where the paths of the particle through the two slits are only imperfectly distinguishable. The following analysis is closely similar to that of Englert where he derives the wellknown Englert-Greenberger-Yasin duality relation [11]. We define the distinguishability of the two paths by

$$
\mathcal{D}=\sqrt{1-\left|\left\langle d_{1} \mid d_{2}\right\rangle\right|^{2}}
$$

where $\left|d_{1}\right\rangle$ and $\left|d_{2}\right\rangle$ are assumed to be normalized, but not necessarily orthogonal to each other. Clearly, for completely orthogonal $\left|d_{1}\right\rangle$ and $\left|d_{2}\right\rangle, \mathcal{D}=1$, and for identical $\left|d_{1}\right\rangle$ and $\left|d_{2}\right\rangle, \mathcal{D}=0$. If $\left|d_{1}\right\rangle$ and $\left|d_{2}\right\rangle$ are orthogonal to each other, one can find an observable of the detector, for which the two states can give two distinct eigenvalues.
Measuring such an observable, one can find out which of the two slits the particle went through.

If the two states are not completely orthogonal, one can write the state $\left|d_{2}\right\rangle$ in terms of a component parallel to $\left|d_{1}\right\rangle$ and a component orthogonal to it

$$
\left|d_{2}\right\rangle=c_{\|}\left|d_{1}\right\rangle+c_{\perp}\left|d_{\perp}\right\rangle
$$

where $\left|d_{\perp}\right\rangle$ is a state orthogonal to $\left|d_{1}\right\rangle$, and $\left|c_{\|}\right|^{2}+\left|c_{\perp}\right|^{2}=1$. Now, suppose one measures an observable which gives different eigenvalues for $\left|d_{1}\right\rangle$ and $\left|d_{\perp}\right\rangle$. If the particle goes through slit 1 , the detector will always give one particular outcome (right answer). However, if the particle goes through slit 2, the detector will give a different value (right answer) with probability $\left|c_{\perp}\right|^{2}$, but will give the same value associated with slit 1 (wrong answer) with probability $\left|c_{\|}\right|^{2}$. So, one cannot distinguish the two paths with certainty if the $\left|d_{1}\right\rangle$ and $\left|d_{2}\right\rangle$ are not orthogonal. One can distinguish the two path with a probability $\left|c_{\perp}\right|^{2}$. Using Equation 4 one can verify that $\left|c_{\perp}\right|^{2}=\mathcal{D}^{2}$. Thus, in this case the probability with which the two paths can be distinguished is equal to $\mathcal{D}^{2}$. In general $\mathcal{D}^{2}$ can be considered to be the likelihood of getting the correct which-way information.

Let us assume that a particle traveling along the $z$ direction passes through a double-slit and also interacts with a which-path detector. We model the particle state as a Gaussian form with width $\epsilon$ when it strikes the slits. Through a process such as the one described by Equation 1, the detector states get correlated with the states of the particle emerging from the two slits. If this happens at time $t=0$, then we can write the combined state of the particle and the which-path detector, in the form

$$
\Psi(x, 0)=A\left(\left|d_{1}\right\rangle e^{-\frac{(x-d / 2)^{2}}{4 \epsilon^{2}}}+\left|d_{2}\right\rangle e^{-\frac{(x+d / 2)^{2}}{4 \epsilon^{2}}}\right)
$$

where $A=\frac{1}{\sqrt{2}}\left(2 \pi \epsilon^{2}\right)^{-\frac{1}{4}}$. We have not given different momenta to the two wave-packets which might result from a "momentum back-action" of the which-way detector. We will show that just the correlation between the two wave-packets and the different states of the recoiling slit is enough to destroy interference. Here we do not expicitly consider the dynamics of the particle in the $z$-direction. We just assume that the wave-packets are moving in the forward direction with an average momentum $p_{0}=h / \lambda$, where $\lambda$ is the d'Broglie wavelength of the particle. Thus the distance $L$ travelled by the particle in a time $t_{L}$ is given by $L=\frac{h}{m \lambda} t_{L}$. This can be rewritten as $\hbar t_{L} / m=\lambda L / 2 \pi$.

After a time $t$, the combined state of the particle and the detector evolves to

$$
\Psi(x, t)=A_{t}\left(\left|d_{1}\right\rangle e^{-\frac{(x-d / 2)^{2}}{4 \epsilon^{2}+2 \hbar t / m}}+\left|d_{2}\right\rangle e^{-\frac{(x+d / 2)^{2}}{4 \epsilon^{2}+2 i \hbar t / m}}\right)
$$


where $A_{t}=\frac{1}{\sqrt{2}}[\sqrt{2 \pi}(\epsilon+\imath \hbar t / 2 m \epsilon)]^{-\frac{1}{2}}$. The probability of finding the particle at position $x$ on the screen is given by

$$
\begin{aligned}
|\Psi(x, t)|^{2}= & \left|A_{t}\right|^{2}\left(e^{-\frac{(x-d / 2)^{2}}{2 \sigma_{t}^{2}}}+e^{-\frac{(x+d / 2)^{2}}{2 \sigma_{t}^{2}}}\right) \\
+ & \left|A_{t}\right|^{2}\left(\left\langle d_{1} \mid d_{2}\right\rangle e^{-\frac{x^{2}+d^{2} / 4}{2 \sigma_{t}^{2}}} e^{\frac{1 x d h t / 2 m \epsilon^{2}}{2 \sigma_{t}^{2}}}\right. \\
& \left.+\left\langle d_{2} \mid d_{1}\right\rangle e^{-\frac{x^{2}+d^{2} / 4}{2 \sigma_{t}^{2}}} e^{-\frac{x x d h t / 2 m \epsilon^{2}}{2 \sigma_{t}^{2}}}\right)
\end{aligned}
$$

where $\sigma_{t}^{2}=\epsilon^{2}+(\hbar t / 2 m \epsilon)^{2}$. Writing $\left\langle d_{2} \mid d_{1}\right\rangle$ as $\left|\left\langle d_{2} \mid d_{1}\right\rangle\right| e^{\imath \theta}$, and putting $\hbar t / m=\lambda L / 2 \pi$, the above can be simplified to

$$
\begin{aligned}
|\Psi(x, t)|^{2}= & 2\left|A_{t}\right|^{2} e^{-\frac{x^{2}+d^{2} / 4}{2 \sigma_{t}^{2}}} \cosh \left(x d / 2 \sigma_{t}^{2}\right) \times \\
& \left(1+\left|\left\langle d_{1} \mid d_{2}\right\rangle\right| \frac{\cos \left(\frac{x d \lambda L / 2 \pi}{4 \epsilon^{4}+(\lambda L / 2 \pi)^{2}}+\theta\right)}{\cosh \left(x d / 2 \sigma_{t}^{2}\right)}\right)(9)
\end{aligned}
$$

Equation 9 represents an interference pattern with a fringe width given by

$$
w=2 \pi\left(\frac{(\lambda L / 2 \pi)^{2}+4 \epsilon^{4}}{\lambda d L / 2 \pi}\right)=\frac{\lambda L}{d}+\frac{16 \pi^{2} \epsilon^{4}}{\lambda d L}
$$

For $\epsilon^{2} \ll \lambda L$ we get the familiar Young's double-slit formula $w \approx \lambda L / d$.

Visibility of the interference pattern is conventionally defined as

$$
\mathcal{V}=\frac{I_{\max }-I_{\min }}{I_{\max }+I_{\min }}
$$

where $I_{\max }$ and $I_{\min }$ represent the maximum and minimum intensity in neighbouring fringes, respectively. In practice, fringe visibility will depend on many things, including the width of the slits. For example, if the width of the slits is very large, the fringes may not be visible at all. Maxima and minima of Equation 9 will occur at points where the value of cosine is 1 and -1 , respectively. The two wave-packets emerge from two narrow slits and quickly expand and overlap because of time evolution. The width of a wave-packet, which was $\epsilon$ initially, is now $\sigma_{t}=\sqrt{\epsilon^{2}+(\hbar t / 2 m \epsilon)^{2}}$ at time $t$. For sufficiently large $\sigma_{t}$, $\cosh \left(x d / 2 \sigma_{t}^{2}\right)$ can be assumed to be $x$-independent over distances of the order of fringe separation. The visibility can then be written down as

$$
\mathcal{V}=\frac{\left|\left\langle d_{1} \mid d_{2}\right\rangle\right|}{\cosh \left(x d / 2 \sigma_{t}^{2}\right)}
$$

Because $\cosh (y) \geq 1$, we get

$$
\mathcal{V} \leq\left|\left\langle d_{1} \mid d_{2}\right\rangle\right|
$$

Using Equation 4 the above equation gives a very important result

$$
\mathcal{V}^{2}+\mathcal{D}^{2} \leq 1
$$

This relation generalizes Bohr's complementarity principle of mutual exclusivity of wave and particles natures, to quantifying the extent to which both these natures can be observed at the same time. It sets a bound on the whichpath distinguishability and the visibility of interference that one can obtain in a single experiment. This inequality was derived earlier by Greenberger and Yasin [10] and Englert [11] in a more general context.

\subsection{Uncertainty and duality}

While it has been argued that the duality relation given by Equation 14 is independent of any kind of uncertainty relation [10,11], there is also another view prevalent in the literature which holds that the process of whichway detection introduces certain uncontrollable phases to the state of the particle, which leads to loss of interference [12,13]. The uncertainty relation is believed to play a role in the latter. Whether complementarity arises out of correlations between the particle and a which-path detector or from the uncertainty principle, has been a subject of some controversy [13-16]. Linked to this controversy is also the question whether the particle receives any momentum kick from the recoiling slit, affecting its interference pattern [17--19]. There have been various approaches to connect complementarity to uncertainty relations [20-23]. We explore this issue in the light of the preceding discussion.

Let us suppose that a particle passing through a doubleslit interacts with a which-way detector, the recoiling slit in our case. Let us suppose that corresponding to particle passing through slits 1 and 2 , the recoiling slit acquires two distinct momentum states. If this is true, we can always find an observable $\hat{P}$ which will give eigenvalues $(1,-1)$ corresponding to the particle passing through slits 1 and $2, \hat{P}\left|p_{1}\right\rangle=\left|p_{1}\right\rangle$ and $\hat{P}\left|p_{2}\right\rangle=-\left|p_{2}\right\rangle$.

In a non-ideal situation, the recoiling slit may have only partial which-way information. Then the states that actually get correlated with the particle paths in Equation 6 could be written as

$$
\left|d_{1}\right\rangle=c_{1}\left|p_{1}\right\rangle+c_{2}\left|p_{2}\right\rangle, \quad\left|d_{2}\right\rangle=c_{2}^{*}\left|p_{1}\right\rangle+c_{1}^{*}\left|p_{2}\right\rangle
$$

With the provision $\left|c_{1}\right|^{2}+\left|c_{2}\right|^{2}=1,\left|d_{1}\right\rangle$ and $\left|d_{2}\right\rangle$ are normalized but not necessarily orthogonal. The ideal situation would correspond to $\left|c_{1}\right|=1, c_{2}=0$ or viceversa, where the detector states would carry full whichway information. For the case $\left|c_{1}\right|=\left|c_{2}\right|=1 / \sqrt{2}$, the detector states would carry no which-way information. Thus, the above form of $\left|d_{1}\right\rangle,\left|d_{2}\right\rangle$ covers all possibilities of mutual overlap.

The square of uncertainty in $\hat{P}$ with any of these two states, is given by

$$
\Delta \hat{P}^{2}=\left\langle\hat{P}^{2}\right\rangle-\langle\hat{P}\rangle^{2}=4\left|c_{1}\right|^{2}\left|c_{2}\right|^{2}
$$




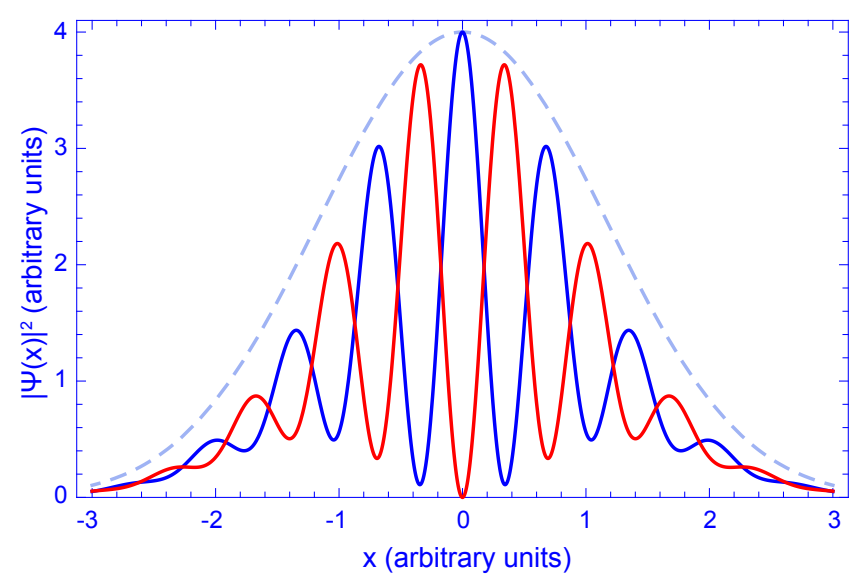

Figure 2: If one correlates the detected particles with measurement results on $\hat{Q}$, one gets two complementary patterns corresponding to $\left|q_{1}\right\rangle$ (blue line) and $\left|q_{2}\right\rangle$ (red line). Without any correlation, there is no intference pattern (dashed line).

Note that distinguishablity, as defined by Equation 4 now has the form

$$
\mathcal{D}^{2}=1-4\left|c_{1}\right|^{2}\left|c_{2}\right|^{2}
$$

This implies that

$$
\mathcal{D}^{2}=1-\Delta \hat{P}^{2}
$$

So, for distinguishablity to be $1, \Delta \hat{P}$ should be zero. If one does not wish to talk in the language of correlations between the particle and the detector carrying which-way information, and just wants to look at interference buildup from individual particles registering on the screen, one has to take into account the change in relative phase of the amplitudes of particle passing through the two slits because of interaction with the which-way detector [19]. This was the approach taken by Bohr in replying to Einstein's recoiling slit experiment. If the particle paths $\psi_{1}(x)$ and $\psi_{2}(x)$ are correlated to the detector states $\left|p_{1}\right\rangle$ and $\left|p_{2}\right\rangle$, so that the combined state is

$$
\Psi(x)=\psi_{1}(x)\left|p_{1}\right\rangle+\psi_{2}(x)\left|p_{2}\right\rangle
$$

there will be a correspondence between detector states and particle states as follows

$$
\begin{aligned}
& \frac{1}{\sqrt{2}}\left(\left|p_{1}\right\rangle+\left|p_{2}\right\rangle\right) \rightarrow \frac{1}{\sqrt{2}}\left[\psi_{1}(x)+\psi_{2}(x)\right] \\
& \frac{1}{\sqrt{2}}\left(\left|p_{1}\right\rangle-\left|p_{2}\right\rangle\right) \rightarrow \frac{1}{\sqrt{2}}\left[\psi_{1}(x)-\psi_{2}(x)\right]
\end{aligned}
$$

Thus, there exists another observable of the recoiling slit, $\hat{Q}$, such that $\hat{Q}\left|q_{1}\right\rangle=\left|q_{1}\right\rangle, \hat{Q}\left|q_{2}\right\rangle=-\left|q_{2}\right\rangle$, and $\left|q_{1}\right\rangle=$ $\frac{1}{\sqrt{2}}\left(\left|p_{1}\right\rangle+\left|p_{2}\right\rangle\right),\left|q_{2}\right\rangle=\frac{1}{\sqrt{2}}\left(\left|p_{1}\right\rangle-\left|p_{2}\right\rangle\right)$. Observables $\hat{Q}$ and $\hat{P}$ obviously do not commute, and both can be represented by Pauli spin operators. For instance, if $\hat{P}=\hat{\sigma_{z}}$, then $\hat{Q}=\hat{\sigma_{x}}$.
In general, the state of a particle passing through the double-slit and interacting with the recoiling slit, can be written in terms of the eigenstates of operator $\hat{Q}$, as follows

$$
\begin{aligned}
\Psi(x)= & \frac{1}{\sqrt{2}} c_{1}\left[\psi_{1}(x)+\psi_{2}(x)\right]\left|q_{1}\right\rangle \\
& +\frac{1}{\sqrt{2}} c_{2}\left[\psi_{1}(x)-\psi_{2}(x)\right]\left|q_{2}\right\rangle
\end{aligned}
$$

with $\left|c_{1}\right|^{2}+\left|c_{2}\right|^{2}=1$. For $\left|c_{1}\right|=\left|c_{2}\right|=\frac{1}{\sqrt{2}}$, and $\psi_{1}, \psi_{2}$ having the form represented in Equation 7, if one correlates the probability of finding the particle on the screen with the measured eigenstate of $\hat{Q}$, Equation 22 yields two shifted interference patterns (see Figure 2). But together, the two patterns kill each other. This is an example of quantum eraser [24], where correlating the detected particle with certain states of the which-way detector can "erase" the which-way information and the interference pattern can be observed.

For any $c_{1}, c_{2}$, the probability of finding the particle on the screen is given by

$$
\begin{aligned}
|\Psi(x)|^{2}= & \frac{1}{2}\left(\left|\psi_{1}(x)\right|^{2}+\left|\psi_{2}(x)\right|^{2}\right) \\
& +\frac{\left|c_{1}\right|^{2}-\left|c_{2}\right|^{2}}{2}\left(\psi_{1}^{*}(x) \psi_{2}(x)+\psi_{2}^{*}(x) \psi_{1}(x)\right)
\end{aligned}
$$

Using the earlier analysis, in particular Equation 13, we can immediately write

$$
\mathcal{V}^{2} \leq\left(\left|c_{1}\right|^{2}-\left|c_{2}\right|^{2}\right)^{2}
$$

The uncertainty in the observable $\hat{Q}$, in the state given by Equation 22, can be evaluated to yield

$$
\Delta \hat{Q}^{2}=1-\left(\left|c_{1}\right|^{2}-\left|c_{2}\right|^{2}\right)^{2}
$$

While doing so, one has to make use of the fact that $\psi_{1}(x)$ and $\psi_{2}(x)$ are orthonormal states [25]. The two equations above yield

$$
\mathcal{V}^{2} \leq 1-\Delta \hat{Q}^{2}
$$

Using Equation 18 and Equation 26 we find

$$
\mathcal{D}^{2}+\mathcal{V}^{2} \leq 2-\left[\Delta \hat{P}^{2}+\Delta \hat{Q}^{2}\right]
$$

For any two-level system, adequately described by Pauli spin matrices, any two spin-components satisfy the following sum uncertainty relation [26]

$$
\Delta \hat{\sigma}_{1}^{2}+\Delta \hat{\sigma}_{2}^{2} \geq 1
$$

which in turn implies that $\Delta \hat{P}^{2}+\Delta \hat{Q}^{2} \geq 1$. Using this result, Equation 27 reduces to

$$
\mathcal{D}^{2}+\mathcal{V}^{2} \leq 1
$$


Thus we find that the Englert-Greenberger-Yasin duality relation also emerges as a consequence of the sum uncertainty relation for certain observables of the recoiling slit.

At this stage it might be useful to make connection between the preceding analysis and Bohr's reply to Einstein. Bohr had argued that a fixed position of the recoiling slit would correspond to a sharp interference. Different fixed positions of the recoiling slit would correspond to slightly shifted interference patterns. Our analysis shows that two distinct values of $\hat{Q}$ lead to two sharp, but mutually shifted, interference patterns (see Figure 2). In our analysis, for an accurate which-way information, one needs an eigenvalue of $\hat{P}$, which will result in a superposition of two values of $\hat{Q}$, and consequently to a superposition of two shifted interference patterns. In Bohr's argument, a distinct value of momentum would lead to a superposition of different positions of the recoiling slit, and a superposition of many shifted interference patterns, and hence, loss of interference. Our $\hat{P}$ and $\hat{Q}$ are analogous to the momentum and position of the recoiling slit, respectively, in Bohr's argument. Thus, the preceding calculation may be viewed as a more quantitative analysis of Bohr's estimate. The Englert-Greenberger-Yasin relation emerges as a more general statement of the Heisenberg uncertainty relation as invoked by Bohr in the context of Einstein's recoiling slit experiment.

\section{Discussion}

The preceding analysis, although applicable to Einstein's recoiling slit experiment, is fairly general. The detector states $\left|d_{1}\right\rangle$ and $\left|d_{2}\right\rangle$ may correspond to states of any other kind of which-way detector. In the analysis of Wooters and Zurek [4], the recoiling slit was modelled as a harmonic oscillator in its ground state, which is a Gaussian state with zero average momentum. The states $\left|d_{1}\right\rangle$ and $\left|d_{2}\right\rangle$ here correspond to Gaussian states with oppositely shifted average momentum. The distinguishability of the two states, as defined by Equation 4 , will put a bound on the visibility of the interference pattern, according to Equation 14

From the preceding analysis we see that interefrence visiblity $\mathcal{V}$ can be 1 only when $\Delta \hat{Q}$ is 0 . Also, the whichpath distinguishability $\mathcal{D}$ can be 1 only if $\Delta \hat{P}$ is 0 . Because $\hat{P}, \hat{Q}$ do not commute, $\Delta \hat{P}, \Delta \hat{Q}$ cannot both be zero at the same time. This can also be assumed to be a fundamental reason enforcing complementarity.

Bohr's argument of the position uncertainty of the recoiling slit did rule out simultaneous observation of interference and obtaining which-path information. This led many to believe that Bohr's complementarity principle was in fact, a tacit restatement of the position-momentum uncertainty relation. However, the sum uncertainty relation for observables $\hat{P}$ and $\hat{Q}$, introduced here, puts a tighter bound on fringe visibility and which-way information. It actually leads to the very fundamental EnglertGreenberger-Yasin duality relation. So, the sum uncertainty of certain two-state observables seems to be enforcing complementarity in a more fundamental way than the Heisenberg uncertainty relation.

From the analysis of subsection 3.2 we have seen that the Englert-Greenberger-Yasin duality relation also comes out from the correlation between the particle paths and which-path detector states. On the other hand, if the particle paths get correlated to certain orthogonal detector states, one can always find two observables $\hat{P}$ and $\hat{Q}$, whose sum-uncertainty relation will be a quantitative statement of complementarity. Thus we see that the mutual exclusivity of wave and particle nature emerges as a consequence of quantum correlation of the particle with the which-way detector states, and also from the sum uncertainty relation of certain observables of the which-way detector. This indicates that uncertainty relations are as much an inherent part of the which-way detection process, as are the quantum correlations. So, quantum correlations and quantum uncertainty relation are two alternate ways of looking at the same phenomenon. Both lead to the fundamental Englert-Greenberger-Yasin duality relation.

Lastly, we point out that there has been a prevailing view that Bohr's reply to Einstein implied that the particle receives momentum kicks due to its interaction with the detector, and that enforces complementarity. We emphasize that Bohr never talked about any momentum backaction on the particle from the recoiling slit. He only said that the particles originating from a particular position (position of the recoiling slit) will lead to a particular position of the interference pattern. A shifted position of the recoiling slit, will lead to a shifted pattern. If there is an uncertainty in the position of the recoiling slit, it will lead to an uncertainty in the location of the fringes, and hence washing out of interference. In our analysis of subsection 3.3, the two eigenstates of $\hat{Q}$ lead to two different locations of the interference pattern (see Figure 2). So, $\hat{Q}$ in our analysis plays the role of position of the recoiling slit in Bohr's argument. Here interference loss is due to different relatives phases associated with the two particle paths, corresponding to different eigenstates of $\hat{Q}$. Any momentum back-action on the particle is an additional baggage, not essential to explaining the loss of interference. 


\section{Acknowledgements}

This work was concieved during the International Workshop on Quantum Information, held on February 20-26, 2012 at the Harish-Chandra Research Institute in Allahabad, India. The authors thank the organisers for providing a platform for exchange of ideas.

\section{References}

[1] Bohr N. The quantum postulate and the recent development of atomic theory. Nature 1928; 121 (3050): 580-590. http://dx.doi.org/10. $1038 / 121580 a 0$

[2] Feynman RP, Leighton RB, Sands M. The Feynman Lectures on Physics, Volume 3. Addison-Wesley, 1966, pp. 1-1.

[3] Bohr N. Discussions with Einstein on epistemological problems in atomic physics. In: Albert Einstein: Philosopher-Scientist, Schilpp PA (editor), Evanston: Open Court Publishing Company, 1949, pp.200-241. http://dx . doi . org/10. 1016/S1876-0503(08)70379-7

[4] Wootters WK, Zurek WH. Complementarity in the double-slit experiment: Quantum nonseparability and a quantitative statement of Bohr's principle. Physical Review D 1979; 19 (2): 473-484. http: //dx.doi.org/10.1103/PhysRevD.19.473

[5] Bertet P, Osnaghi S, Rauschenbeutel A, Nogues G, Auffeves A, Brune M, Raimond JM, Haroche S. A complementarity experiment with an interferometer at the quantum-classical boundary. Nature 2001; 411 (6834): 166-170.http://dx.doi.org/ $10.1038 / 35075517$

[6] Utter RS, Feagin JM. Trapped-ion realization of Einstein's recoiling-slit experiment. Physical Review A 2007; 75 (6): 062105. http: //dx . doi . org/10. 1103/PhysRevA. 75.062105

[7] von Neumann J. Mathematical Foundations of Quantum Mechanics. Princeton University Press, 1955.

[8] Scully MO, Englert B-G, Walther H. Quantum optical tests of complementarity. Nature 1991; 351 (6322): 111-116. http://dx.doi.org/10. $1038 / 351111 \mathrm{a} \theta$

[9] Schrödinger E. Discussion of probability relations between separated systems. Mathematical Proceedings of the Cambridge Philosophical Society 1935; 31 (4): 555-563. http://dx . doi . org/10.1017/ S0305004100013554
[10] Greenberger DM, Yasin A. Simultaneous wave and particle knowledge in a neutron interferometer. Physics Letters A 1988; 128 (8): 391-394. http://dx.doi.org/10.1016/ 0375-9601(88)90114-4

[11] Englert B-G. Fringe visibility and which-way information: an inequality. Physical Review Letters 1996; 77 (11): 2154-2157.http://dx .doi.org/ 10.1103/PhysRevLett. 77.2154

[12] Tan SM, Walls DF. Loss of coherence in interferometry. Physical Review A 1993; 47 (6): 4663-4676. http://dx.doi.org/10.1103/ PhysRevA.47.4663

[13] Storey P, Tan S, Collett M, Walls D. Path detection and the uncertainty principle. Nature 1994; 367 (6464): 626-628. http://dx.doi.org/10. $1038 / 367626 a 0$

[14] Englert B-G, Scully MO, Walther H. Complementarity and uncertainty. Nature 1995; 375 (6530): 367368. http://dx . doi .org/10.1038/375367b0

[15] Wiseman H, Harrison F. Uncertainty over complementarity? Nature 1995; 377 (6550): 584. http: //dx.doi.org/10.1038/377584a0

[16] Barad K. Meeting the Universe Halfway: Quantum Physics and the Entanglement of Matter and Meaning. Duke University Press, 2007.

[17] Wiseman HM. Directly observing momentum transfer in twin-slit "which-way" experiments. Physics Letters A 2003; 311 (4-5): 285-291. http://dx, doi . org/10.1016/SQ375-9601(Q3)00504-8

[18] Dürr S, Nonn T, Rempe G. Origin of quantummechanical complementarity probed by a "whichway" experiment in an atom interferometer. Nature 1998; 395 (6697): 33-37. http://dx.doi.org/ $10.1038 / 25653$

[19] Unnikrishnan CS. Origin of quantum-mechanical complementarity without momentum back action in atom-interferometry experiments. Physical Review A 2000; 62 (1): 015601. http: //dx . doi . org/10 1103/PhysRevA.62.015601

[20] Björk G, Söderholm J, Trifonov A, Tsegaye T, Karlsson A. Complementarity and the uncertainty relations. Physical Review A 1999; 60 (3): 18741882.http://dx .doi .org/10.1103/PhysRevA. 60.1874 
[21] Marzlin K-P, Sanders BC, Knight PL. Complementarity and uncertainty relations for matterwave interferometry. Physical Review A 2008; 78 (6): 062107. http://dx.doi.org/10.1103/ PhysRevA.78.062107

[22] Huang J-H, Zhu S-Y. Complementarity and uncertainty in a two-way interferometer.http://arxiv. org/abs/1011.5273

[23] Bosyk GM, Portesi M, Holik F, Plastino A. On the connection between complementarity and uncertainty principles in the Mach-Zehnder interferometric setting. http://arxiv.org/abs/1206.2992

[24] Scully MO, Drühl K. Quantum eraser: A proposed photon correlation experiment concerning observation and "delayed choice" in quantum mechanics. Physical Review A 1982; 25 (4): 2208-2213. http: //dx.doi.org/10.1103/PhysRevA.25.2208

[25] Hari Dass ND, Qureshi T, Sheel A. Minimum uncertainty and entanglement. International Journal of Modern Physics B 2013; 27: 1350068. http: //arxiv.org/abs/1107.5929

[26] Hofmann HF, Takeuchi S. Violation of local uncertainty relations as a signature of entanglement. Physical Review A 2003; 68 (3): 032103. http:// dx.doi.org/10.1103/PhysRevA.68.032103 